\title{
Evolutionary Cognitive Economics
}

\author{
Gilles Paquet \\ Centre on Governance \\ University of Ottawa
}

\begin{abstract}
A bstract
The paper reviews that limitations of the neo-classical economics paradigm in dealing with information: focus on individual rationality, static view of the world, unduly parsimonious characterization of the world of cognition. Then it sketches the contours of an alternative paradigm built on selective rational ity, imperfect mental representations, cognitive structures as filters, and an adoption/adaptation dynamics: selection mechanisms adopting certain representations, rationales and conventions, but rules and patterns also adapting to the evolving milieu. The new evolutionary cognitive paradigm is shown to be pregnant with new insights into the structure and functioning of modern economies but also likely to generate some reformulation in our policy prescriptions
\end{abstract}

Classification code:

Key words: information, knowledge, cognition, learning, evolution

Gilles Paquet is Professor of E conomics and Public $M$ anagement at the Faculty of A dministration and Director of the $C$ entre on G overnance at the $U$ niversity of $O$ ttawa, 136 J ean-J acques L ussier, Ottawa, Ontario, Canada K 1N 6N5. He can be reached by phone at 613-562-5800 ext. 4729, by fax at 613-562-5164, and his e-mail address is paquet@ admin.uottawa.ca 
"W hat is now needed is a far-from-equilibrium information economics which allows for innovation, evolution, and learning".

Max Boisot

Introduction

An economic system is a set of conversations, rationales, protocols, conventions, organizations and institutions providing the coordination and the orientation maps to ensure a viable process of production, allocation and distribution of goods, services and information for a population. It can also be defined as the communication system that underpins this process of coordination of production and exchange.

It is one of the great merits of 'information economists' to have generated a greater awareness (1) of the extent to which economic systems have been transformed, over the last few decades, from arrangements centered on the production, allocation and distribution of material goods to arrangements with a focus on knowledge production, and (2) of the extraordinarily crude simplifications that the conventional neo-classical paradigm has imposed on its analyses of the economy as communication system. M achlup (1962), Porat (1977) and others have shown that there has been a dematerialization of economic activity; Hayek (1945), Shackle (1949), Simon (1955), Boulding (1956) and others have demonstrated in quite different ways how inadequate the neo-classical approach has been in its treatment of the knowledge base of modern economies. 
Our objective in this note is to build on this second strand of argument in order to show that whatever heuristic power the neo-classical paradigm may have had in other times, it has become inadequate to deal with contemporary realities. In the first section, we will indicate briefly why this is the case. In the following section, we outline the contours of a heuristically powerful alternative paradigm based on cognitive and evolutionary economics. We then proceed in section 3 to show how this alternative paradigm reveals new terrains and suggests new prescriptions. In conclusion, we bemoan the fact that it is unlikely that this paradigm will yield its full complement of insights in the near future: the neo-classical paradigm is a sacred cow and putting it out to pasture may take a little while.

1. The limitations of the conventional paradigm

Perfect information has been a traditional assumption of neo-classical economics. Market economies have been presumed to be composed of autonomous mildly self-reflective individuals optimizing their objective function subject to constraints, and these individuals have been assumed to know what they wanted and to know their environment. Nobody bothered to inquire as to where that knowledge was coming from (Piore 1995:100). N umerous theorems were constructed on these foundations showing that, in a perfectly competitive world, these omniscient agents would be induced to take action that would lead to a social optimum making the highest and best use of existing resources. 
$M$ any have been uneasy about this triplet of assumptions (1) perfect information, (2) perfect competition, and (3) a focus on allocative efficiency within a fairly static $\mathrm{N}$ ewtonian economic world (Boisot 1995:11). This condition has generated two sets of responses: one has been bent on exorcising some of the unreasonableness of the first two assumptions without damaging the overall N ewtonian economics edifice; while the other has been geared to constructing a viable al ternative paradigm to the Newtonian edifice that allocative efficiency concerns have built.

In the first case, this has led to the blooming of frictional economics: a variety of scenarios to get around the pure and perfect competition assumption (from the transaction costs world of Coase $(1937,1960)$ and Williamson $(1975,1985)$ to the contestable markets of Baumol et al. (1982) but also to thirty years of exploration of a world where perfect information might not prevail (when information is costly, asymmetric, etc.) and therefore calling for slight amendments to the traditional analytical apparatus to accommodate the ensuing friction.

In the second case, this has led to a variety of alternative weltanschauungen: from Hayek (1945) and the numerous economists working in the A ustrian tradition (see O' D riscoll and Rizzo 1985 for a good overview), and their emphasis on the role of knowledge and discovery, to the exploration of cognitive structures (Boulding 1956), to the abandonment of the Newtonian equilibrium models altogether in favor of the study of adaptive or Schumpeterian efficiency and chaotic evolutionary processes (Leydesdorff and van den Besselaar 1994). 
Despite these responses, the neo-classical paradigm still reigns supreme in the economics profession. Even the recent attempts at building syncretic information economics perspectives, by the admission of their authors (M CCain 1992; L amberton 1994; Boisot 1995; Castells 1996), have not produced 'the new viable paradigm' likely to be adopted as a new vision of the world by the economics profession.

This is ascribable to a variety of reasons. First, the immense amount of intellectual capital invested in the traditional paradigm, and the most effective protective belt and 'dynamic conservatism' generated by the very exclusive process of hiring, promotion and publication that exists within the economics profession (Katouzian 1980) have made it very difficult for anyone who is not a member of the tribe to achieve any position of authority in the most influential economics departments. Second, there has been an unfortunate tendency, even among the very best information economists, to avoid confrontation with the traditional tribe and to continue to regard information as "a commodity" al beit "only to a limited extent" (L amberton 1994); this has been the case even though Lamberton and others recognized that "economic activity is a process, its participants having both histories and contexts", and that "progress lies in learning" (our emphasis). If this is the case, communication and information cannot simply be reduced to the level of physical inputs (Paquet 1994) and informational resources allocated like physical resources. Third, there has been a fundamental misunderstanding generated by the focus on information processing rather than on knowledge production in the development of the alternative paradigm. This has sterilized much of the work in information economics for attention has been focused exclusively on explicit information flows processed by rational actors, while much of 
cognition is buried in tacit knowledge. This has derailed information economics away from the problem of cognition (Baumard 1996).

Y et, despite those blockages, three fundamental challenges have been mounted against the ruling paradigm, and would appear not to have been countered:

(1) there may not be a need to build our view of the world, as the neo-classical paradigm does, on autonomous individual rational decision-making: first, because of the fact that there are numerous reasons to believe that this "irrational passion for rationality" is most unrealistic; and second, because of the fact that the most simplistic irrational behavior might yield the traditional results through simple natural selection processes (Perlman 1990);

(2) the focus on the pursuit of static efficiency in the use of existing resources (that has prevailed in the neo-classical paradigm) is at odd with the central concern for social learning as a way to progressivity (i.e., the right evolving mix of exploration of new processes and exploitation of existing processes) that underpins modern economies (de la M othe and Paquet 1996);

(3) the link between an actors' or a group's representation of the world and contextual reality may not be as unproblematic as is presumed by the neo-classical paradigm: all sort of rationales, cognitive structures, conventions and institutions are mediating in fundamental ways the relationship between agents and context, and are in many ways more meaningful units of analysis than either agents, organizations or context. 
2. The evolutionary cognitive paradigm

These flaws in the traditional paradigm (an undue focus on individual instrumental rationality, an unduly static view of the world, and an unduly parsimonious characterization of the complex world of cognition) would appear to warrant our exploring other avenues.

The challenges that the new paradigm must face are

(1) to ensure that the centrality of cognition as a neural and social process is factored into the analysis, and that there is full appreciation that the process of cognition is neither unbounded nor costless nor unbiased;

(2) to ensure that a realistic appreciation of the structures of the socio-economy organized around knowledge production replaces the antiquated industrial-age images of the socio-economy organized around energy and material resources; and

(3) to ensure that one can explicate the dynamic joint processes of exploration and exploitation in organizational learning and in the evolution of the institutional order.

In the next two sub-sections, we describe (a) the novel approach suggested by cognitive theory, and (b) the new mechanisms unveiled by evolutionary theory. We then show in the third subsection how these might be combined into a new evolutionary cognitive economics paradigm. 
a. Cognitive economics examines the process of extraction of information from the environment through perception, and the development of knowledge through communication. In this framework, information exists to the extent that it becomes embodied in the brain as a pattern or in an artifact (capital) designed to act as a surrogate for the brain. Instead of focusing on the allocation of existing informational resources, cognitive economics focuses on the production of new knowledge. To the extent that cognition is conditioned and restricted by the mechanism of brain and mind, it is all but impossible to understand how information and communication matter without a fair idea of the way in which people gain knowledge. This is the main reason for cognitive economics to focus on the protocols leading to cognition.

The brain registers sensory inputs. These inputs are information extracted by perceptual systems. In the beginning is the percept. The perceptual systems search the environment for behaviorally relevant change, and extract information patterns from the invariants in the environment. This information is then registered in the brain in the form of neuronal nets (i.e., of interactive assemblages of neurons in patterns). A new experience creates a pattern recognition cascade often built from existing patterns plus a small set of new patterns generated as new neurons fire in response to input. A non-trivial message therefore triggers a modification, a reframing or reconfiguration of existing patterns though the firing of new neurons (Coward 1990).

Cognitive economics focuses on cognition as the source of meaningful, i.e., behaviorally-relevant information, and aims at developing a higher ratio of meaningful information to noise through an improvement of the various information/communication enhancement mechanisms: more effective 
skills at extracting patterns, easier transformation of frames of reference, reduction of cognitive dissonance, etc.

But cognition is neither unbounded nor costless nor unbiased. Leibenstein and Simon have done much to show that human reasoning proceeds through selective search by applying rules of thumb, and that such a search stops when a satisfactory solution is found (W eiermair and Perlman 1990; Simon 1992). This sort of search builds on experience, and therefore is conditioned by the existence of frameworks that evolve over time both through interaction with the environment and with other agents. This family of readinesses or interpretative frames is a series of filters that stand between agents and their environment. These frames may either accelerate or decelerate a capacity for an individual to learn from experience (Ciborra 1990). Such frameworks may be culturally determined, but they are not invariant. They evolve as a result of experience and context through selection and mutation.

Knowledge is not only objective and social. It is often subjective and idiosyncratic. There are forms of connoisseurship or tacit knowledge that may be non-communicable but may allow one to develop a winning strategy (Baumard 1996). This sort of knowledge may be the result of implicit learning or the embodiment of much social practice. It takes the form of a sort of flair that one may not be able to explain, but that is nevertheless useable knowledge.

Cognition does not occur only at the individual level. Creative individuals can rarely reach their goals in isolation. They operate in the context of organizations embodying a communication 
network. Organizations al so learn from experience. Experiences with real-world groups (H utchins 1991) have shown that new circumstances or breakdown/loss of perceptual equipment, generate new stable work configurations that are not unlike a modified neuronal network.

There are also interactions between organizational learning and cognitive structures: framing a challenge as a puzzle means that it will be receiving some attention, while framing it as an anomaly may simply mean that it will be ignored and rationalized away. In the first case, learning is probable; in the second case, most unlikely (Kahneman and Tversky 1979).

b. Evolutionary economics is built on the assumption of limited cognitive capabilities for both humans and organizations. This entails a continuous process of trial and error. A nd since the cost of thinking is not zero, once some appreciation of context has been arrived at, routines, conventions, and standard procedures are adopted that would appear to ensure survival or satisficing performance.

These routines, rooted in context and history, are embedded in a pattern of rationales, rules, conventions, and institutions that provide the process of decision-making and learning with unity and stability. In this world, the meaningful units of analysis are the parallel patterns of belief systems and mental representations and techno-organizational conventions and rules in which the process is vested.

A t any time, these representations and rules may be more or less fitting, i.e., they may be more 
or less effective socio-technical armistices between the evolving physical environment and the evolving values and plans of agents and groups. The degree of fitness is not invariant as circumstances change: it is rooted in the probability of survival and in the capacity to develop the requisite competences and capabilities to survive.

Given the always imperfect nature of the mental representations, and of the conventions and rules, and the mistake-ridden learning process, the modification of both representations and rules constitutes the way in which the socio-economy evolves, transforms and learns. This learning may be more or less effective, depending on the nature of the challenges generated by the environment, and the nature of the competences, readinesses and reactive capacities of agents and organizations. For instance, the pattern of representations and rules may easily accommodate minor variations in the environment and adapt quickly to these new circumstances. However, it may only be capable of limited learning within a narrow band of circumstances. In the face of radical changes in the environment that call for a dramatic reframing of representations, rules and conventions, it may mainly generate a great deal of cognitive dissonance and dynamic conservatism, and be incapable of learning (Ciborra 1990:210).

Indeed, as N elson and W inter have shown (1982), organizations tend to stick to their usual routine as long as performance remains above certain target levels. It is only when performance indicators fall below such levels that the organization searches for better alternatives. This balance between the exploitation of the available knowledge and the exploration and search for new knowledge and new possibilities [underlined by $M$ arch (1991)] is closely related to the mechanisms of selection 
and mutation: a procedure or routine that performs well being adopted by the system (i.e., a higher probability of survival being bestowed on it) in the case of selection, while some misfit between routines and the changing milieu may lead to a mutation in the routine or procedure (D osi and M arengo 1994).

Evolution emerges from this process of mutual learning between agents and organizations, in the form of the parallel and interactive processes of selection and mutation. This learning may be faster or slower depending on the nature of the organization. For instance, hierarchical organizations may be able to filter out new local events and prevent learning of certain sorts: the design of organizations most efficient for knowledge exploitation purposes may lead to learning disabilities in exploration, to organizations that have lesser exploratory competences.

Toulmin has sorted change mechanisms into four categories (Toulmin 1981). Calculative change is triggered by rational choice as employed by mainstream economic theory; homeostatic change occurs in response to stimuli in accordance with fixed rules, as in single-loop learning in an organization (using new means); development change is typified by life-cycle theories and might correspond to instances of double-loop learning (learning new goals), i.e., to the restructuring of the selecting unit, its goals or mission; finally, populational change is triggered by changes in the environment and in the adoption of selective units (i.e., a change in its probability of being adopted and nurtured by the environment) - natural selection. 
A $n$ adaptation-adoption model built entirely on the sole calculative and populational forces of change is somewhat simplistic. Individuals more or less deliberately create institutions and organizations that evolve, and inject homeostatic and development changes. The nature of organizations, and the modification of their rules of functioning, may dramatically affect the direction and speed of the learning process: new information may either feed or inhibit evolution, and the cognition process may accelerate or decelerate the process of change. Indeed, one may even have to unlearn in order to be able to progress. Evolution is not unbounded, but evolutionary epistemology is already a blossomming field capable of clarifying these boundaries (H ahlweg and Hooker 1989).

c. The concerns raised over the last century by the neo-classical paradigm have generated a large number of efforts at constructing meaningful alternative paradigms. $M$ any of these efforts have been carried out under the general flag of a refurbished cognitive approach (Piore 1992) and others under the label of an evolutionary approach (Witt 1993). However, most of those efforts, despite much imagination, have been unable to shake off more than a few of the shackles with which the neo-classical paradigm is burdened. A reasonable and workable alternative to the traditional paradigm might be what we call the evolutionary cognitive paradigm, (ECP) built on a more realistic appreciation of both cognition and the evolutionary processes. 
In a summary way,

1. the ECP posits a complex and turbulent environment;

2. it also assumes bounded and selective rationality and imperfect mental representations;

3. a great importance is given to cognitive structures as filters in the cognition process;

4. the unit of analysis is the pattern or the net of relationships: the cognitive representations, technical arrangements and organizational routines within which mistake-ridden individual and organizational learning occurs;

5. the governing relation is the fitness of pattern and milieu;

6. the dynamics stems from a mixture of milieu selection mechanisms adopting the most viable patterns, representations, rationales, rules and conventions on the one hand, and network mutation activities through which the rationales, rules and patterns adapt to the evolving milieu;

7. this sort of adaptation/adoption dynamics operates at many levels, and in different segments of the network space, and it is the resulting mix of specialization and integration that ensues transversally that distils the institutional order;

8. the institutional order is the set of orientation maps guiding the coevolution of patterns, networks, absorptive capacity, capabilities and competences with the regimes in force in the contextual environment.

There is a degree of congruence between the neural net character of the cognitive structures and the neural net character of the learning organizational/institutional networks. In both cases, learning is akin to the transformation of the pattern. Both the neural network and the social 
networks are self-organizing information systems. Indeed, both the cognitive space and the organizational/institutional space are self-organizing neural net patterns of the kind to be found in a living brain, where the nodes are neurons or nerve cells, connected both locally and over long distance by fibrous dendrites and axons, and are organized in layer structures.

This connectionist model suggests that "a layered system of many signal-processing units, interconnected and interacting in parallel within and between layers, has some remarkable properties" (Ziman 1991:74). By allowing each node to respond to incoming signals, a neural net can learn pattern recognition. F or neither the cognition nor the organizational/institutional process are passively receiving information: they are actively pattern searching. Our mind invents and imposes patterns where none existed previously. The same may be said about the organizationalinstitutional network: "parallel regions in the network search for similar patterns, some of which can be combined to demonstrate the presence or absence of higher order patterns" (Coward 1990:59).

It is in the invention of those higher order patterns that creativity lies. Both in our cognitive and organizational spaces, different neural nets extract different patterns and combine them to produce and integrate recognizable wholes. The mind and the organization recreate the whole or invent a new whole: learning is about pattern extraction capability, and about the ways in which one can integrate in parallel or in a cascading way the diversity of patterns extracted by different regions of the brain or of the organization. Evolutionary development is no quantum leap: it is a smooth progression of developing complexity by adding connections and cascading patterns to the 
cognitive or organizational space (Coward 1990:76).

To build the ability to analyze and respond to radically different types of experience requires a transformation of the patterns, a creation of new 'regions', the mobilization of 'unused neurons' and the development of new connection sensitivities (1) to support patterns-extraction experiences that are analogous to those the brain or organization has experienced but reframed somewhat, perceived in a slightly different way, and (2) to generate pattern cascades from previously disparate regions of experiences, to make new cascade patterns (Coward 1990; Ziman 1991).

\section{New terrains, new prescriptions}

The evolutionary cognitive paradigm, based on individual and organizational learning in a world of bounded and selective rationality, pattern thinking, pattern causality, self-organization and evolutionary change, opens new vistas and forces the revision of many prescriptions. This is not the place for a comprehensive list of such new insights. But a few examples of new terrains open to investigation, and of new prescriptions might help to fix ideas about the heuristic power of the new paradigm.

a. Chief among the new terrains open to exploration by the new paradigm are three areas pregnant with new insights into the structure and functioning of modern economies. 
First, one must single out the world of inert areas, selective rationality, cognitive filters and cognitive failures, that has been probed and analyzed in the mildly heterodox literature over the last decades (L eibenstein 1976; Kahneman and T versky 1979; A kerl of and Dickens 1982; M cC ain 1992). Their surprising results have been interpreted as a string of anomalies. Consequently, they were either ignored or rationalized away.

$Y$ et those results have opened a new frontier in economics and in public policy. They invite an analysis of the power of mediating cognitive structures (Elster 1989; F rey 1990) and an inquiry into areas like the cognitive basis of entrepreneurship, the search for novelty, creativity, and impulse-filtering in organizations.

In all those areas that have been somewhat neglected by neo-classical economics, the evolution of belief systems and cognitive structures has become a subject of more attentive analyses. They have also become the focus of public policy in a way that had been anticipated by Simon and Leibenstein: cognitive economics has simply been intent on extending and radicalizing many of their ideas on cognition.

Second, the whole area of norms, conventions, routine patterns and other organizational features (the organizational equival ent of cognitive structures) has also become of central importance. The pattern of relationships is the new unit of analysis: this is the world of rationales, technoorganizational patterns, networks and moral contracts. They are embodied in neural- network-type relationships that are very similar to those that embody knowledge in the brain: learning or the 
production of new knowledge takes the form of a new pattern of relationships, and social learning means embodiment in a new set of relationships (N orth 1996).

The reconfiguration of the net, either by the addition of one node or by the modification of its structure through a change in the valence of one node or the connections with it, is the vehicle of change and therefore of collective learning (F avereau 1994).

Third, the new centrality of patterns of relationships cannot but bring to the center of the stage the challenge of understanding the dynamics of evolutionary self-organized economic systems.

These issues have al ready caught the attention of even those most determined to work within the neo-classical paradigm (Schelling 1978; Leydesdorff and Van Den Besselaar 1994; Krugman 1996). While this new approach in terms of self-organization is nothing but another name for network learning, it suggests a new interest in network externalities, M yrdalian cumulative processes, dynamic increasing returns, cumulative technologies, and emergent properties of complex systems.

This constitutes a world quite different from the N ewtonian world of neo-classical economics. It is a world full of non-linearities, where old-fashioned predictability is becoming much more difficult, if not completely, and where emergent properties are the new rule. M oreover, the ECP provides for interesting ways to take into consideration biased representations and cognitive structures as major elements in the shaping of path-dependent long-run trends ( $\mathrm{N}$ orth 1996). 
b. This new paradigm is bound to force much reformulation in our analyses and policy prescriptions.

First, the new paradigm focuses on rules of thumb, or routines or patterns that are rooted in belief systems and cognitive structures and embedded in a social context. It is therefore impossible to presume that individuals accultured in certain ways, or whose circumstances are different, would elect the same strategies. Economics needs to address language and culture as filters, and the socio-cultural context as an important component in the emergence of certain rationales, conventions and rules. This might help to gauge the impact of cultural and belief systems (noted by H ampden-T urner and T rompenaars 1993) and the social capital effects (noted by Putnam 1995) on the institutional order and on economic performance (N orth 1996).

In this search for an interpretation of belief systems and context, 'technical rationality' is not as helpful a guide as positivists have suggested. It leads to a "dilemma of rigor and relevance": it fails to provide useable knowledge to practitioners as long as the analysts pretend to stay outside the concreteness of experience. The analysts are forced to track down pattern causality in their investigation of collective learning: they work like a plumber, tracing back "a leak to its source ... through a chain of possible causes ... at different locations in the system ... on the basis of a background knowledge of the system which is commonplace and largely tacit" (Schon 1995:83).

Policy action in such a context has to deal directly with the cognitive structures ands belief systems, with the cultural filters, with the perception of the diffuse pattern causality and even with 
'social capital' as a way of addressing the problem of economic performance, rather than counting exclusively on modifying the incentive reward system of monads (Tussman 1977).

Second, the requirement to shift the focus of analysis away from idealized individuals to patterns of action, routines, etc., is bound to shift much of the analyses toward a probing of the cognitive and learning styles of organizations in an effort to understand and to influence the cumulative learning process that is at the source of good economic performance and progressivity.

Organizations, like individuals, have patterns of cognition and learning. Case studies have documented their differential capacity to navigate the whole field of individual and collective, and explicit and tacit knowledge (represented as a $2 \times 2$ table): the particular type of knowledge management and learning strategy adopted (its capacity to make sequentially the highest and best use of the four types of knowledge -- individual/explicit, individual/tacit, collective/explicit and collective/tacit) explains the success or failure of an organization (Baumard 1996). There is no unique path through this $2 \times 2$ matrix any more than a set of rigid sequential rules for a surfer: much depends on the context and on the particular capabilities of the organization.

Baumard's case studies of the strategic management of knowledge by Indigo, Qantas, Pechiney and Indosuez have revealed that difficult and ambiguity-laden crises are resolved at the mesolevel, at the level of the 'community of practice', that actors develop attitudes calling for tacit alliances and informal relations, and that their actions use repertories of routines that are in good currency in the organization and rely on local collective knowledge (Baumard 1996:195). 
Third, the recognition of the immense importance of tacit knowledge in individual and social learning has led to much interest in the possibility of designing loci of conversation and creativity equipped to make the highest and best use of this reservoir of knowledge. In a rapidly evolving, surprise-generating context, organizations are the locus of on-going conversations that will produce new knowledge and value added through networking and partnering. And these conversations are more fruitful at the level of the 'community of practice'. That is the central message of Wikstrom and Normann (1994): the value-creating process is the result of coproduction through interlinkages and conversations among all partners. The main challenge then is to determine what is the best way to organize knowledge production if the objective is to generate an organization characterized by learning and innovation.

Corporate culture embodies generally unwritten principles meant to generate a relatively high level of coordination at low cost by bestowing identity and membership (K reps 1990). This corporate culture is nested at the organization level in the central features of work practice: stories of flexible generality about events of practice that act as repositories of accumulated wisdom, the evolution of these stories constituting collective learning of an evolving way to interpret conflicting and confusing data, but also an on-going social construction of a community of interpretation.

It is not easy to construct a robust corporate culture. It is fundamentally a matter of trust. Trust is at the core of the fabric of the communities of practice. Trust is a way to transform "laborers into members", to convert an employment contract into a membership contract: "the concept of 
membership, when made real, would replace the sense of belonging to a place with a sense of belonging to a community" (Handy 1995). A nd belonging is one of the most powerful agents of mobilization. One may therefore expect a redefinition of work as conversation, as sharing stories, as becoming a member of a community of practice as suggested in the recent writings of Peters (1994) and Handy (1995).

\section{Conclusion}

But no reform of economics should be expected overnight. "For the reformer has enemies in all those who profit by the old order, and only lukewarm defenders in all those who would profit by the new order, this lukewarmness arising partly from fear of their adversaries, who have the laws in their favour; and partly from the incredulity of mankind, who do not truly believe in anything new until they have had actual experience of it" (M achiavelli (orig. 1537) 1952:49-50) It is only when economists have developed, on the one hand, a much greater awareness of the results generated by cognitive science over the last decades, and, on the other hand, a much greater appreciation of the complexity of the socio-political nexus of organizations and institutions that one may hope that a new synthesis might emerge.

Some progress has already been made on the second front. There is some recognition of the need to enrich the neo-classical paradigm with a better appreciation of organizational and institutional issues (L anglois 1986). There are al ready journals and forums where these issues are debated. On 
the first front, little progress has been made up to now because of the mental block surrounding rational decision-making as an icon. But the M CCain synthesis in 1992 and the recent Nobel lecture of Douglass North in 1993 may indicate that the time for a breakthrough may be near (McCain 1992; N orth 1996).

M oreover, evolutionary economic models have explored a relatively large array of situations in which both cognitive and organizational issues have been explicitly taken into account. And they have begun to do so in a formalized manner in places like the J ournal of E volutionary E conomics. This is not unimportant. As K rugman noted, in the present context of the economics profession, " an academic idea flourishes best if it is expressed in a rather technical way, even if the technical difficulty is largely spurious" (K rugman 1996:16). So one may expect that some versions of the ECP may become widely disseminated within the economics profession over the next decade.

But the likely scenario is that the evolutionary cognitive paradigm will subsist in the underground until such a time as one cause célèbre is found that demonstrates quite dramatically that the new paradigm generates dramatically different policy recommendations in a domain of acute public concern. Then, and only then, can there be a hope to see a "representational redescription" (as North might call it).

But this cautiously optimistic scenario may be unrealistic, for many such occasions have presented themselves and have been missed. The classic book of $\mathrm{N}$ elson and $\mathrm{W}$ inter did not succeed in having anything but a succès d'estime in 1982, even though it proposed new insights for the 
explanation of the productivity slowdown of the 1970s that had stumped the profession. A nd the authority of the 'freshwater economists' has remained untainted even after decades of important counter-arguments to their prescriptions on issues of the utmost importance for advanced economies. One must therefore not underestimate the formidable resilience of the some of those neo-classical ideas perpetrated by defunct economists.

$\mathrm{GP} /$

References

A kerlof, G.A. and W.T. Dickens, 1982, The economic consequences of cognitive dissonance. A merican Economic Review 72 (3), 307-319.

Baumard, P. 1996, Organisations déconcertées: la gestion stratégique de la connaissance. (M asson, Paris).

Baumol, W., J. Panzar and R. Willig. 1982. Contestable markets and the theory of industry structure (H arcourt B race J ovanovich, New Y ork)

Boisot, M. 1995, Information space ( Routledge, L ondon)

Boulding, K.E., 1956. The image (The U niversity of M ichigan Press, A nn A rbor) 
Castells, M. 1996, The rise of the network society (Blackwell, Oxford)

Coborra, C.U., 1990, X-efficiency, transaction costs, and organizational change. In: Weiermair, K. and M. Perlman (Eds) Studies in economic rationality - X-efficiency examined and extolled: Essays written in the tradition of and to honor Harvey Leibenstein (The U niversity of M ichigan Press, Ann Arbor), 205-222.

Coase, R., 1937, The nature of the firm, Economica, 4, 386-405.

Coase, R., 1960, The problem of social cost, J ournal of $L$ aw and Economics 3 (1), 1-44.

Coward, L.A. 1990, Pattern thinking (Praeger, Westport, Conn.)

de la Mothe, J. and G. Paquet (Eds) 1996, Evolutionary economics and the new international political economy, (Pinter, London)

Dosi, G. and L. Marengo, 1994, Some elements of an evolutionary theory of organizational competences. In: England, R.W. (Ed) Evolutionary concepts in contemporary economics (The University of M ichigan Press, A nn A rbor), 157-178.

Elster, J. 1989, N uts and bolts for the social sciences (Cambridge University Press: Cambridge). 
Favereau, 0. 1994, Règle, organisation et apprentissage collectif: un paradigme non-standard pour trois théories hétérodoxes. In: Orléan, A., (E d.) 1994, A nalyse économique des conventions ( Presses U niversitaires de France, Paris), 113-137.

Frey, B.S., 1990, Human behavior: ipsative and objective possibilities. In: Weiermair, K. and M. Perlman (E ds) Studies in economic rationality - X-efficiency examined and extolled: Essays written in the tradition of and to honor Harvey Leibenstein (The U niversity of M ichigan Press, A nn Arbor), 71-93.

Hahlweg, K. and C.A. Hooker (E ds) 1989, Issues in evolutionary epistemology (State U niversity of New Y ork Press, Albany).

Hampden-Turner, C. and A. Trompenaars 1993, The seven cultures of capitalism (Currency/D oubleday, N ew Y ork)

Handy, C. 1995, Trust and the virtual organization, Harvard Business Review 73 (3), 40-50.

Hayek, F.A. von 1945, The use of knowledge in society, A merican Economic Review 35 (4), 519-530.

Hutchins, E., 1991, Organizing work by adaptation, Organization Science 2 (1), 14-39. 
Kahneman, D. and A. Tversky, 1979, Prospect theory: an analysis of decision under risk, Econometrica 47 (2), 263-291.

Katouzian, H., 1980, Ideology and method in economics, (N ew Y ork U niversity Press: N ew Y ork).

Kreps, D.M., 1990, Corporate culture and economic theory. In: Alt, J.E. and K. A. Shepsle (E ds) Perspectives on Positive Political Economy, (Cambridge U niversity Press, Cambridge) 90143.

Krugman, P.R., 1996 The self-organizing economy (Blackwell: Oxford)

Lamberton, D.MCL., 1994, The information economy revisited. In: Babe, R.E. (Ed.) Information and communication in economics (K luwer A cademic Publishers, Boston), 1-33.

Langlois, R.N. (Ed.), 1986, Economics as process - Essays in the new institutional economics (CambridgeU niversity Press, Cambridge)

Leibenstein, H., 1976, Beyond economic man (Harvard University Press, Cambridge).

Leydesdorff, L. and P. van den Besselaar (Eds) 1994, Evolutionary economics and chaos theory (Pinter: London). 
M achiavelli, N., 1952 (orig. 1537) The Prince (M entor Book, N ew Y ork)

M achlup, F., 1962, The production and distribution of knowledge in the U nited States, (Princeton University Press, Princeton).

March, J.G., 1991, Exploration and Exploitation in Organizational Learning, Organization Science 2 (1), 71-87.

McCain, R.A., 1992, A framework for cognitive economics (Praeger, Westport, Conn.)

Nelson, R.R. and S.G. Winter, 1982, An evolutionary theory of economic change (Harvard University Press, Cambridge)

North, D.C., 1996, Economic performance through time. In:. Alston, L.J. Eggertsson, T. and North, D.C. (Eds) Empirical studies in institutional change (Cambridge University Pres: Cambridge) 342-355.

O'D riscoll, G.P. and M .J. Rizzo (Eds) 1985, The economics of time and ignorance, (Blackwell, Oxford). 
Paquet, G. 1994, From the information economy to evolutionary cognitive economics. In: Babe, R.E. (Ed.) Information and communication in economics (Kluwer Academic Publishers, Boston), 34-40

Perlman, M., 1990, The evolution of X-efficiency theory. In: Weiermair, K. and M. Perlman (E ds) Studies in economic rationality - X-efficiency examined and extolled: Essays written in the tradition of and to honor Harvey Leibenstein (The University of M ichigan Press, A nn A rbor), 725.

Peters, T. 1994, Crazy times call for crazy organizations (V intage, N ew Y ork).

Piore, M.J., 1992, Fragments of a cognitive theory of technological change and organizational structure. In: Nohria, N. and R.G. Eccles (Eds), N etworks and organizations (Harvard Business School Press, Boston), 430-444.

Piore, M.J. 1995, Beyond individualism (Harvard University Press, Cambridge)

Porat, M.U. 1977, The information economy (U.S. Government Printing Office, Washington)

Putnam, R.D., 1995, Bowling alone: A merica's declining social capital, Journal of Democracy $6(1), 65-78$. 
Schelling, T., 1978, M icromotives and macrobehavior (N orton, N ew Y ork).

Schon, D.A., 1995, Causality and causal Inference in the study of organizations. In: Goodman, R.F. and W.R. Fisher (Eds) Rethinking knowledge (State U niversity of N ew Y ork, A lbany) 69101.

Shackle, G.L.S., 1949, Expectations in economics ( The U niversity Press, Cambridge)

Simon, H.A., 1955, A behavioral model of rational choice, Quarterly J ournal of Economics, 59, $99-118$.

Simon, H.A., et al., 1992, Economics, bounded rationality and the cognitive revolution (Edward Elgar, A ldershot )

Toulmin, S., 1981, Human adaptation. In: Jensen, U. and R. Harre (Eds) The philosophy of evolution (St M artin's Press, New Y ork)

Tussman, J., 1977, Government and the mind (Oxford University Press, N ew Y ork)

Wikstrom, S. and R. Normann 1994. Knowledge and V alue (Routledge, L ondon).

Williamson, O.E., 1975, M arkets and hierarchies (The Free Press, N ew Y ork) 
Williamson, O.E. 1985, The economic institutions of capital ism (The F ree Press, N ew Y ork)

Witt, U. (Ed.) 1993, Evolutionary economics (Edward Elgar, A ldershot)

Ziman, J. 1991, A neural net model of innovation Science and Public Policy 18 (1), 65-75. 